\title{
Antioxidant consumption and repletion kinetics in nasal lavage fluid following exposure of healthy human volunteers to ozone
}

\author{
I.S. Mudway*, A. Blomberg**, A.J. Frew**, S.T. Holgate ${ }^{+}$, T. Sandström*, F.J. Kelly*
}

\begin{abstract}
Antioxidant consumption and repletion kinetics in nasal lavage fluid following exposure of healthy human volunteers to ozone. I.S. Mudway, A. Blomberg, A.J. Frew, S.T. Holgate, T. Sandström, F.J. Kelly. C ERS Journals Ltd 1999.

ABSTRACT: To obtain information on the real-time events occurring within human respiratory tract lining fluids (RTLFs) during ozone exposure, sequential nasal lavage was performed on 13 human volunteers exposed on separate occasions to 0.2 parts per million $\mathrm{O}_{3}$ and filtered air (2-h exposures, with intermittent exercise).

Nasal lavage was performed and blood samples obtained at four time points throughout each exposure: pre-exposure (Pre-E), $1 \mathrm{~h}$ into exposure (1h-E), immediately postexposure (0h-PE) and $1 \mathrm{~h}$ post-exposure (1h-PE). Endobronchial mucosal biopsies were obtained at $1.5 \mathrm{~h}$-post exposure (1.5 h-PE).

Nasal RTLF neutrophilia was not apparent during, or $1.5 \mathrm{~h}$ after, $\mathrm{O}_{3}$ exposure. Furthermore, activation of the pre-existing neutrophil population did not occur. Airway permeability was not altered by this $\mathrm{O}_{3}$ exposure regimen. Sequential lavage resulted in significant washout of RTLF ascorbic acid, reduced glutathione, extracellular superoxide dismutase and myeloperoxidase at $1 \mathrm{~h}-\mathrm{E}, 0 \mathrm{~h}-\mathrm{PE}$ and $1.5 \mathrm{~h}-\mathrm{PE}$ relative to baseline Pre-E values. In contrast, RTLF uric acid (UA), total protein and albumin concentrations did not display washout kinetics. Of the antioxidants examined, only UA was clearly depleted by $\mathrm{O}_{3}$, concentrations, falling by $6.22 \mu \mathrm{mol} \cdot \mathrm{L}^{-1}$ at 1h-E, compared with $1.61 \mu \mathrm{mol} \cdot \mathrm{L}^{-1}(\mathbf{p}<0.01)$ during control air exposure. The establishment of a new pseudo-steady-state concentration of RTLF UA $(70 \%$ of Pre-E values) during the second hour of $\mathrm{O}_{3}$ exposure was coincident with a small but significant increase in plasma $U A$ concentration $\left(19.27\left(O_{3}\right)\right.$ versus $1.95 \mu \mathrm{mol} \cdot \mathrm{L}^{-1}$ (air), $\mathbf{p}<\mathbf{0 . 0 5}$ ).

These data demonstrate that inhalation of 0.2 parts per million $\mathrm{O}_{3}$ results in the depletion of nasal respiratory tract lining fluid uric acid and that this regional loss of uric acid leads to a small increase in plasma uric acid concentration. Whilst the reaction of uric acid with inspired $\mathrm{O}_{3}$ may confer protection locally, the role of upper airway uric acid as a sink for inhaled $\mathrm{O}_{3}$ is not supported by these findings.

Eur Respir J 1999; 13: 1429-1438.
\end{abstract}

*Lung Biology, Cardiovascular Research, The Rayne Institute, St Thomas' Hospital, London, UK. **Dept of Respiratory Medicine and Allergy, University Hospital and the National Institute for Working Life, Umeå, Sweden. ${ }^{+}$Air Pollution Group/Immunopharmacology Group, University Medicine, Southampton General Hospital, Southampton, UK.

Correspondence: F.J. Kelly

Cardiovascular Research

The Rayne Institute

St Thomas' Hospital

London SE1 7EH

UK

Fax: 441719280658

Keywords: Air pollution

glutathione

lung

nasal lavage

oxidative stress

uric acid

Received: August 261998

Accepted after revision February 11999

This study was supported by the Swedish Asthma and Allergy Association, the Swedish Work Environmental Fund, the Swedish Heart and Lung Foundation and the Medical Research Council (UK)

Human respiratory tract lining fluids (RTLFs) are well invested with a range of small molecular weight antioxidants and antioxidant enzymes [1-3]. This defensive network is ideally located to protect the respiratory epithelium, representing the first physical interface encountered by inspired agents, from the damaging effects of inhaled oxidants [4]. Indeed, the fact that insoluble gases such as ozone and nitrogen dioxide are unlikely to cross this aqueous compartment unreacted [4-6] suggests that their ultimate effects should be interpreted in the context of their initial interactions with RTLF constituents [7]. The products arising from interactions with protein and lipid components are thought to be harmful, capable of acting as toxic intermediates and resulting in epithelial injury and inflammation. Reactions with antioxidants, however, are thought of as protective, limiting the extent of these former interactions [7].

The distribution of antioxidants within the RTLF varies greatly throughout the airways, with uric acid (UA) predominating in the upper airways [2] and reduced glutathione (GSH) appearing more important at distal sites
[8]. This regional variation may have major implications for the protective role played by these moieties in ameliorating the damaging effects of inhaled oxidants such as $\mathrm{O}_{3}$. For example, $\sim 40 \%$ of inhaled $\mathrm{O}_{3}$ is removed from the airstream in the extrathoracic airways [9], where there is a high concentration of UA, an antioxidant known to be highly reactive toward $\mathrm{O}_{3}[10,11]$.

In this study, it was hypothesized that the interaction of $\mathrm{O}_{3}$ with components of the nasal RTLF (particularly UA) might serve two important protective functions, not only conferring protection locally but also protecting the more distal lung by scrubbing $\mathrm{O}_{3}$ from the inhaled airstream and, hence, limiting the dose of $\mathrm{O}_{3}$ reaching these regions. To test this hypothesis, sequential nasal lavage (NL) was employed on a group of healthy human subjects, exposed on separate occasions to 0.2 parts per million (ppm) $\mathrm{O}_{3}$ and filtered air. NL was employed to sample upper airway RTLF as it represented a relatively noninvasive procedure, which could be repeated at multiple time points. This approach allowed antioxidant and inflammatory responses, occurring within nasal RTLFs, to be followed during and 
after the exposure period. As stated in the accompanying article in this issue [12], the authors believe that events occurring within the initial period of interaction between $\mathrm{O}_{3}$ and the RTLFs are critical to an understanding of the ultimate symptomatic responses observed after $\mathrm{O}_{3}$ exposure.

Consequently, the aims of this study were two-fold. The first aim was to demonstrate the extent to which environmentally relevant $\mathrm{O}_{3}$ concentrations impact upon the nasal RTLF antioxidant screen. This information could then be used to establish how the kinetics of $\mathrm{O}_{3}$-induced antioxidant consumption, observed ex vivo [13] are modified by repletion mechanisms in vivo. Plasma samples were also obtained at parallel sampling points to determine the extent to which events conceptually confined to the RTLF impacted upon the remote plasma pool. The second aim was then to relate any significant interactions occurring within the upper airway RTLFs to the symptomatic responses seen in these subjects: pulmonary decrements observed immediately post-exposure (0h-PE) and the mucosal inflammatory and distal lung RTLF redox, inflammatory and permeability responses observed at $1.5 \mathrm{~h}$ post-exposure (1.5h-PE).

\section{Subjects and methods}

\section{Chemicals}

All chemicals used in the following protocols were obtained from either Sigma Chemical Company (Poole, UK) or Merck (Poole, UK), unless otherwise stated.

\section{Subjects and study design}

Detailed subject demographics and the experimental protocol are given in the accompanying article [12], and are summarized only briefly here. Thirteen subjects were recruited into the study, according to the criteria previously outlined, five female and eight male, median age 23 yrs (range 20-31 yrs). Subjects were exposed on two separate occasions, once to filtered air, and once to 0.2 ppm $\mathrm{O}_{3}$. Consecutive exposures were separated by $\geq 3$ weeks and subjects were assigned to these using a randomization method to ensure that an equal number of individuals underwent the air and $\mathrm{O}_{3}$ treatments as their first exposure. Exposures lasted $2 \mathrm{~h}$, with subjects performing a standard alternate $15 \mathrm{~min}$ exercise (sufficient loading to produce a mean minute ventilation of 20 $\mathrm{L} \cdot \mathrm{min}^{-1} \cdot \mathrm{m}^{-2}$ body surface area) and rest protocol [14]. The study was conducted in a single-blind, crossover control fashion. Peripheral blood was withdrawn and NL performed at four time points throughout the exposures: preexposure (Pre-E), $1 \mathrm{~h}$ into exposure (1h-E), immediately post- (0h-PE) and $1 \mathrm{~h}$ post-exposure (1h-PE). The final NL procedure was performed immediately after final lung function testing, and prior to premedication of the subjects for the bronchoscopy procedure at $1.5 \mathrm{~h}-\mathrm{PE}$.

\section{Nasal lavage procedure and treatment of recovered fluid}

NL samples were collected according to the method of HARDER et al. [15]. Briefly, 10 aliquots $(0.1 \mathrm{~mL}$ each $)$ of phosphate-buffered saline (PBS) were sprayed into each nostril and the recovered material transferred into a sterile plastic receptacle. The procedure was repeated five times, i.e. a total volume of $5 \mathrm{~mL}$ was sprayed into each nostril. The container was then immediately placed on ice and the recovered aspirate filtered through a sterile $100-\mu \mathrm{m}$ pore nylon filter to remove mucus aggregates before centrifugation at $400 \times g$ for $15 \mathrm{~min}\left(4^{\circ} \mathrm{C}\right)$ to isolate the cellfree fraction. The cell-free supernatant was then frozen in aliquots at $-80^{\circ} \mathrm{C}$ prior to analysis. NL samples for malondialdehyde (MDA) determination were treated with synthetic antioxidants prior to storage, $10 \mu \mathrm{L}$ of a solution containing $2 \mathrm{mM}$ butylated hydroxytoluene (BHT) and 2 $\mathrm{mM}$ Deferoxamine mesylate (DM) was added per $\mathrm{mL}$ of recovered NL fluid (i.e. final concentration $20 \mu \mathrm{mol} / \mathrm{L}$ ). The cell pellet was then resuspended in $1 \mathrm{~mL}$ PBS, and total and differential cell counts performed as outlined in the accompanying article [12] with respect to bronchial wash and bronchoalveolar lavage (BAL) fluid cell determinations.

\section{Treatment of plasma samples}

Peripheral blood, $10 \mathrm{~mL}$, was collected at each of the prescribed time points and immediately divided into two equal aliquots in heparinized tubes maintained on ice, one of which contained $50 \mu \mathrm{L}$ of $2 \mathrm{mM}$ BHT, $2 \mathrm{mM}$ DM. The two whole blood aliquots were then centrifuged at 2,000 $\times$ $g$ for $10 \mathrm{~min}$ at $4{ }^{\circ} \mathrm{C}$. The resultant plasma was then aliquoted as required prior to storage at $-80^{\circ} \mathrm{C}$. Plasma samples protected with BHT and DM were used for the determination of the oxidative damage markers, MDA and protein carbonyls, while those without supplemental antioxidants were used for the determination of endogenous antioxidant concentrations.

\section{Antioxidant, oxidative damage marker and soluble me- diator determinations}

NL fluid was assayed for a range of antioxidant moieties (GSH, ascorbic acid (AA), UA, $\alpha$-tocopherol, and extracellular superoxide dismutase (EC-SOD)), oxidation markers (MDA and glutathione disulphide (GSSG)) and soluble mediators (total protein, albumin and myeloperoxidase (MPO)). The following markers were also determined in plasma: antioxidants (AA, UA, $\alpha$-tocopherol, and total thiol), and oxidation markers (MDA and protein carbonyls). GSH and GSSG were measured according to the method of BAKER et al. [16]. NL fluid and plasma AA and UA concentrations were determined simultaneously using reverse phase-high performance liquid chromatography (RP-HPLC) with electrochemical detection [17], and $\alpha$-tocopherol using RP-HPLC with detection via absorbance at $292 \mathrm{~nm}$ [18]. Plasma total sulphydryls were measured according to the protocol described by WAYNOR et al. [19]. EC-SOD in NL fluid was quantitated using a double antibody sandwich enzyme-linked immunosorbent assay [20], MPO using a radioimmunoassay kit (Kabi Pharmacia Diagnostics, Uppsala, Sweden). Total protein and albumin were measured using the bicinchoninic acid method of SMITH et al. [21] and a commercially available kit from Boehringer Mannheim (Mannheim, Germany) 
respectively. Finally, with respect to oxidation markers, HPLC determination of MDA was based on the method of CHIRICO [22], previously described in detail [23], and protein carbonyls according to the method of REZNICK and PACKER [24].

\section{Data analysis}

To ensure that rejection of the null hypothesis was not simply an artefact of multiple testing, the primary hypothesis was limited to detecting changes (positive or negative) in nasal RTLF UA, AA and GSH concentrations. All other data presented here are "exploratory" in nature and conclusions drawn from these data sets weighted accordingly. In all cases rejection of the null hypothesis was accepted at the 5\% level, using a two-tailed test of significance.

Parameters determined in plasma were found to be predominately parametric in character (Shapiro-Wilks normality test). Data are therefore expressed throughout as mean \pm SD. Primary analysis to confirm significant differences within plasma data sets were therefore conducted using a two-way repeated measures analysis of variance (ANOVA) with factors for sampling time and exposure group. All comparisons between groups were conducted using the Student-Newman-Keuls method. Where these preliminary analyses indicated significant differences between exposures at various sampling times, the absolute molar difference across the interval (air and $\mathrm{O}_{3}$ ) versus Pre$\mathrm{E}$ values was determined and comparison between the molar change under the two exposure conditions performed using a paired t-test.

In contrast, NL fluid parameters (including derived partition ratios) were nonparametrically distributed and could not be easily transformed to yield parametric values. NL fluid data are therefore expressed throughout as medians with 25th and 75th percentile ranges. Analysis of NL fluid data sets were conducted using the nonparametric Quade two-way ANOVA, with pairways comparison between groups (time and treatment) using the t-distribution. As with plasma parameters, where the initial analysis revealed significant changes caused by the exposure conditions, the absolute molar change across the pertinent time intervals (versus Pre-E concentration) was determined, and air versus $\mathrm{O}_{3}$ concentration changes compared using Wilcoxon's signed-rank test.

Details of correlation analyses are discussed at greater length in the relevant Results subsection; the expressions used are outlined in the Appendix. Briefly, all analyses were conducted using the Spearman rank-order correlation, and were limited to an examination of the NL and plasma UA results. Correlations were performed to ascertain whether NL UA responses were predictive or causally related to the plasma response. In addition, NL responses were correlated with the significant pulmonary and inflammatory responses reported in the accompanying article [12]. Results of analyses are expressed as the correlation coefficient (Spearman's rho, $\rho$ s) with associated significance level. Correlation analyses were only performed using those parameters which had been shown to be significantly perturbed by $\mathrm{O}_{3}$ exposure. Possible links between associations were assessed using partial correlation analysis. All statistical analyses were performed using the

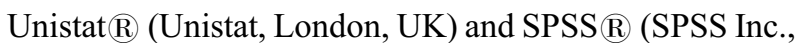
Chicago, IL, USA) statistical packages on a Windowsbased PC platform.

\section{Results}

\section{Nasal lavage}

The NL procedure was well tolerated by all subjects, with an overall median NL fluid recovery of 60.0 (47.5$65.0) \%$ for all lavages performed $(\mathrm{n}=104)$. In addition, no evidence of a significant effect of air versus $\mathrm{O}_{3}$ was noted on lavage fluid recovery. Similarly, repeated lavage procedures did not affect recovered volume. All data in the text and figures are based on concentrations expressed per unit volume of recovered NL fluid.

Baseline antioxidant and oxidative damage marker concentrations in nasal lavage fluid

Two (air and $\mathrm{O}_{3}$ ) Pre-E NL fluid samples were obtained from each subject. Antioxidant concentrations in these PreE samples ( $\mathrm{n}=13)$ were: UA, $29.2(18.1-38.1) \mu \mathrm{M}$ (air) and $22.7(16.1-30.7) \mu \mathrm{M}\left(\mathrm{O}_{3}\right)$; AA, 1.7 (1.4-2.7) and 1.9 (1.32.9) $\mu \mathrm{M}$; GSH, $0.8(0.6-1.3)$ and $1.7(07-4.4) \mu \mathrm{M}$; and EC-SOD, $2.2(1.5-2.6)$ and $2.0(1.4-2.3) \mathrm{ng} \cdot \mathrm{mL}^{-1}$. Sufficient NL fluid to determine EC-SOD was also available in 11 of the 13 subjects. $\alpha$-Tocopherol was only observed in a small proportion (30\%) of the samples, and then at much lower concentrations than the water soluble antioxidants: $26.1(0.0-158.5) \mathrm{nM}$ (air) and $30.3(0.0-58.6)$ $\mathrm{nM}\left(\mathrm{O}_{3}\right)$. Two oxidation markers, MDA and GSSG, were also determined in NL fluid. The latter was largely below the detectable limits for the majority of samples, both in Pre-E and exposed samples. In contrast, MDA was detected in the majority $(80 \%)$ of samples, Pre-E concentrations being $15.0(6.8-27.0) \mathrm{nM}$ (air) and $20.0(0.0-33.9)$ $\mathrm{nM}\left(\mathrm{O}_{3}\right)$. Of the Pre-E determination, only pre-air and pre- $\mathrm{O}_{3}$ GSH concentrations differed significantly $(\mathrm{p}=$ 0.02). Despite this quantitative difference in Pre-E GSH values, a significant association between an individual's Pre-E GSH concentration was apparent $\left(\rho_{\mathrm{s}}=0.6, \mathrm{p}<0.05\right)$, suggesting that the pattern of an individuals GSH concentration was constant with time.

The Pre-E concentrations quoted above are derived from samples obtained from all 13 subjects. Prior to analysing the responses of these moieties to air and $\mathrm{O}_{3}$ exposure, individuals with anomalous Pre-E concentrations ( $>1.5$ times the interquartile range outside the 75 th percentile, and not stable for the individual with time) were excluded. This precaution was taken to prevent spuriously large changes, which would bias the overall group response, occurring in the air or $\mathrm{O}_{3}$ exposure arms. Where both Pre-E values were outliers (and were quantitatively similar), the subject was kept in the analysis, as a previous study had shown good intrasubject stability of NL fluid antioxidant determinations over the time interval employed in this study [25].

\section{Nasal lavage antioxidant responses to $\mathrm{O}_{3}$ exposure}

UA was present in all NL fluid samples, irrespective of sampling time or exposure conditions. Two subjects (sets 
of paired data) were excluded from the analysis of $\mathrm{O}_{3}$ versus air responses as one of their Pre-E values was a clear outlier. UA concentrations at each time point and for both exposures are summarized in figure 1 for the 11 individuals used in the full analysis. Using this reduced data set, a significant loss of NL UA was observed $1 \mathrm{~h}-\mathrm{E}$ to 0.2 ppm $\mathrm{O}_{3}$, falling $-6.22(-15.9-1.9) \mu \mathrm{M}$ compared with $-1.61(-2.8-5.7) \mu \mathrm{M}(\mathrm{p}<0.01)$ in the control air exposure. UA concentrations remained depressed during the second hour of $\mathrm{O}_{3}$ exposure $(\mathrm{p}<0.05)$ compared with parallel control values, but did not fall further from the concentrations observed $1 \mathrm{~h}-\mathrm{E}$, remaining at $\sim 70 \%$ of Pre-E concentrations. 1h-E UA concentrations although still depressed in the $\mathrm{O}_{3}$ exposure arm were no longer significantly different from air values. Notably, no washout of UA was observed as a consequence of repeated NL

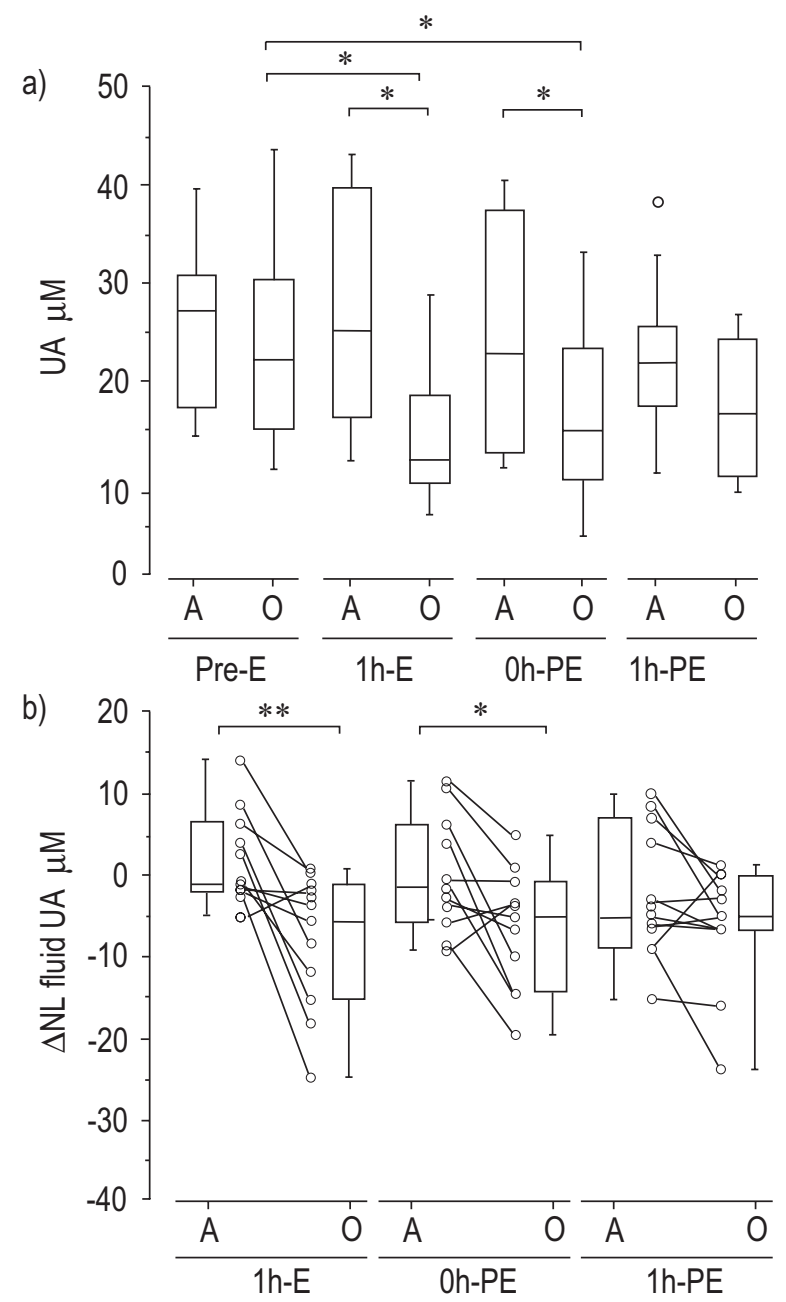

Fig. 1. - Response of nasal respiratory tract lining fluid uric acid (UA) to 0.2 parts per million $\mathrm{O}_{3}(\mathrm{O})$ and control air $(\mathrm{A})$ in healthy human subjects: a) UA concentration at each sampling time point and exposure condition; and b) Absolute change in plasma UA concentration for each of the sampling time points versus pre-exposure (Pre-E) values. Data are summarized in the form of box plots (median, 25th and 75th percentiles and $\pm 1.5 x$ interquartile range with one individual outlier shown $(\bigcirc)$ ). Statistical analyses were performed using Quade two-way analysis of variance (ANOVA) for nonparametrically distributed data $(n=11)$, with multiple comparisons using the t-distribution. Air and $\mathrm{O}_{3}$ responses across each time interval were compared using the Wilcoxon signed rank test. $1 \mathrm{~h}-\mathrm{E}$ : $1 \mathrm{~h}$ into exposure; $0 \mathrm{~h}-\mathrm{PE}$ : immediately post-exposure; $1 \mathrm{~h}-\mathrm{PE}$ : $1 \mathrm{~h}$ post-exposure; $\Delta$ : change. $*$ : $\mathrm{p}<0.05 ; * *$ : $\mathrm{p}<0.01$. procedures, although a slight downward trend was observed overall, from 27.7 (17.8-31.3) $\mu \mathrm{M}$ Pre-E (air) to $22.3(17.8-25.94) \mu \mathrm{M}$ at $1 \mathrm{~h}-\mathrm{PE}$.

As with UA, AA was present in all NL samples analysed, although concentrations were only $7 \%$ of the former. In contrast to UA, considerable washout of AA occurred during the sequential lavages performed during both air and $\mathrm{O}_{3}$ exposures, levels falling by $47.8(39.5-112.0)$ and 36.8 (24.1-69.3)\% respectively (table 1$)$. Thereafter, concentrations in both air and $\mathrm{O}_{3}$ exposure groups did not alter further, but remained depressed compared with Pre$E$ values. Notably, no direct loss of AA attributable to $\mathrm{O}_{3}$ was observed over and above that lost through washout alone. Similar washout kinetics were observed for both EC-SOD and GSH under both air and $\mathrm{O}_{3}$ exposure conditions. Whilst $\mathrm{O}_{3}$ did not affect EC-SOD concentrations, some evidence of an $\mathrm{O}_{3}$-dependent effect on $\mathrm{GSH}$ was noted at $0 \mathrm{~h}-\mathrm{PE}$. This effect was not significant when the whole data set was considered, largely because of the variation between pre-air and $\mathrm{O}_{3}$ concentrations. However, when the absolute changes in concentration Pre-E versus 0h-PE were compared, a small additional loss of GSH owing to $\mathrm{O}_{3}$ was observed: $-0.4(-0.8-0.03)$ versus $-1.0(-1.6--0.2) \mu \mathrm{M}(\mathrm{p}=0.03)$. This difference was, however, no longer apparent at $1 \mathrm{~h}-\mathrm{PE}$.

Airway permeability and oxidative damage marker responses

Changes in nasal epithelial permeability as a consequence of $\mathrm{O}_{3}$ exposure were assessed by determining NL fluid total protein and albumin concentrations; the data are summarized in table 1 . Similar to UA, no washout of either marker was observed as a consequence of sequential lavage. Further, $\mathrm{O}_{3}$ appeared to have no effect on either marker, suggesting that epithelial permeability remained unaltered despite exposure to this oxidant gas. Oxidation of lipid moieties present within the nasal RTLF was determined by means of NL fluid MDA concentrations. Although MDA was detected in the majority of NL fluid samples at nanomolar concentrations, it did not increase as a consequence of $\mathrm{O}_{3}$ exposure (data not shown).

\section{Airway inflammatory responses}

Differential leukocyte counts revealed that $>98 \%$ of all nasal RTLF cells were neutrophils. Therefore, only changes in the total number of nasal RTLF neutrophils in response to $\mathrm{O}_{3}$ were considered in detail. Neutrophil numbers were used to follow potential $\mathrm{O}_{3}$-induced airway neutrophilia, whilst the concentration of MPO was utilized as a marker of neutrophil activation. The results are summarized in table 2. No consistent neutrophil response to either air or $\mathrm{O}_{3}$ exposures was observed. A transient, though nonsignificant, increase in cell number occurred at $1 \mathrm{~h}-\mathrm{E}$ and $0 \mathrm{~h}-\mathrm{PE}$ with air exposure, but numbers had fallen significantly below the Pre-E value at $1 \mathrm{~h}-\mathrm{PE}$. In contrast, no change in the number of neutrophils was observed at any time point within the $\mathrm{O}_{3}$ exposure arm of the study. Significantly fewer neutrophils were observed following $\mathrm{O}_{3}$ exposure at $0 \mathrm{~h}-\mathrm{PE}$ compared with air values. However, given the difference in Pre-E cell numbers pre-air and $\mathrm{O}_{3}$ exposure, this difference is viewed as 
Table 1. - Nasal lavage (NL) fluid antioxidant and protein responses to air and 0.2 parts per million $\mathrm{O}_{3}$ exposure

\begin{tabular}{|c|c|c|c|c|c|}
\hline \multirow{2}{*}{ Parameters } & \multirow{2}{*}{ Exposure } & \multicolumn{4}{|c|}{ Time point } \\
\hline & & Pre-E & $1 \mathrm{~h}-\mathrm{E}$ & Oh-PE & 1h-PE \\
\hline \multirow[t]{2}{*}{ Ascorbic acid $^{+} \mu \mathrm{M}$} & Air & $2.1(1.4-3.0)$ & $1.2(0.6-1.6)^{*}$ & $1.1(0.4-1.4)^{*}$ & $1.0(0.5-1.2)^{*}$ \\
\hline & $\mathrm{O}_{3}$ & $1.8(1.2-2.6)$ & $0.8(0.4-0.9)^{*}$ & $0.6(0.5-1.0)^{*}$ & $0.7(0.6-1.4)^{*}$ \\
\hline \multirow[t]{2}{*}{$\mathrm{GSH}^{*} \mu \mathrm{M}$} & Air & $0.8(0.4-1.2)$ & $0.3(0.2-0.8)$ & $0.4(0.3-0.9)$ & $0.2(0.1-0.5)^{*}$ \\
\hline & $\mathrm{O}_{3}$ & $1.6(0.6-3.6)$ & $1.2(0.5-1.3)$ & $0.6(0.2-0.9)^{*}$ & $0.5(0.1-1.1)^{*}$ \\
\hline \multirow[t]{2}{*}{$\mathrm{EC}-\mathrm{SOD}^{\#} \mathrm{ng} \cdot \mathrm{mL}^{-1}$} & Air & $2.2(1.5-2.6)$ & $1.7(1.2-2.7)^{*}$ & $1.5(0.8-2.2)^{*}$ & $1.1(0.8-1.2)^{*}$ \\
\hline & $\mathrm{O}_{3}$ & $2.0(1.4-2.3)$ & $1.4(1.2-1.9)^{*}$ & $1.3(1.2-2.0)^{*}$ & $1.2(0.7-1.4)^{*}$ \\
\hline \multirow[t]{2}{*}{ Total protein $\mathrm{mg} \cdot \mathrm{mL}^{-1}$} & Air & $0.52(0.39-0.68)$ & $0.39(0.34-0.43)$ & $0.39(0.26-0.46)$ & $0.47(0.36-0.53)$ \\
\hline & $\mathrm{O}_{3}$ & $0.44(0.34-0.58)$ & $0.27(0.21-0.38)$ & $0.30(0.24-0.43)$ & $0.26(0.22-0.46)$ \\
\hline \multirow[t]{2}{*}{ Albumin $\mathrm{mg} \cdot \mathrm{mL}^{-1}$} & Air & $0.07(0.04-0.09)$ & $0.09(0.09-0.11)$ & $0.11(0.04-0.14)$ & $0.11(0.04-0.15)$ \\
\hline & $\mathrm{O}_{3}$ & $0.05(0.03-0.10)$ & $0.03(0.03-0.08)$ & $0.05(0.03-0.11)$ & $0.07(0.03-0.10)$ \\
\hline
\end{tabular}

Data are presented as median with 25 th and 75th percentiles shown in parentheses. All values are expressed per unit volume of recovered NL fluid $(n=11-13)$. Statistical analysis was performed using the Quade two way analysis of variance (ANOVA), with multiple comparisons using the t-distribution. Rejection of the null hypothesis was assumed at $\mathrm{p}<0.05 .{ }^{+}:$one subject excluded from analysis as pre- $\mathrm{O}_{3}$ value was an outlier $(\mathrm{n}=12) ;{ }^{*}$ : two subjects excluded due to insufficient NL fluid. Pre-E: pre-exposure; $1 \mathrm{~h}-\mathrm{E}$ : $1 \mathrm{~h}$ into exposure; 0h-PE: immediately post-exposure; $1 \mathrm{~h}-\mathrm{PE}$ : $1 \mathrm{~h}$ post-exposure. GSH: reduced glutathione; EC-SOD: extracellular superoxide dismutase. *: significantly different from Pre-E value.

a statistical anomaly, and not indicative of any effect of $\mathrm{O}_{3}$ on this cell population. MPO concentrations in the RTLFs displayed typical washout kinetics, with concentrations falling with successive lavage procedures. No evidence of an $\mathrm{O}_{3}$-induced activation of nasal RTLF neutrophils was noted at any time point following $\mathrm{O}_{3}$ exposure.

\section{Plasma antioxidant responses to $\mathrm{O}_{3}$ exposure}

Plasma antioxidant concentrations were determined PreE. The concentrations for all 13 subjects are summarized below, with pre-air values given first: UA, $249.8 \pm 117.9$ and $295.6 \pm 102.3 \mu \mathrm{M}$; AA, $26.4 \pm 15.3$ and $28.8 \pm 7.3 \mu \mathrm{M}$; plasma total thiol, $0.69 \pm 0.12$ and $0.62 \pm 0.14 \mu \mathrm{M}$; and $\alpha$-tocopherol, $14.2 \pm 5.1$ and $12.4 \pm 3.9 \mu \mathrm{M}$. The concentrations and the wide range of interindividual variation outlined above are in line with previous reports [26]. Notably, Pre-E concentrations of each moiety did not vary significantly from each other over the sampling interval studied. No alteration in the concentrations of AA, total thiol or $\alpha$-tocopherol was observed as a consequence of either exposure. In contrast, at $0 \mathrm{~h}-\mathrm{PE}\left(\mathrm{O}_{3}\right)$, a significant increase $(\mathrm{p}<0.05)$ in plasma UA concentration was observed relative to air concentration over the same interval, a significant difference which was maintained at $1 \mathrm{~h}-\mathrm{PE}$ (fig. 2 ). The absolute change in concentration at these time points relative to Pre-E values was relatively modest:
$+22.1 \pm 20.3\left(\mathrm{O}_{3}\right)$ versus $-1.0 \pm 26.7 \mu \mathrm{M}$ at $0 \mathrm{~h}-\mathrm{PE}(7.5 \%$ of the Pre-E concentration), and $+20.2 \pm 12.9\left(\mathrm{O}_{3}\right)$ versus $+7.1 \pm 13.2 \mu \mathrm{M}$ at $1 \mathrm{~h}-\mathrm{PE}(8.1 \%$ of Pre-E concentration). Importantly, the individual magnitude of these plasma changes were not quantitatively related to the NL UA responses outlined previously.

\section{Plasma oxidative damage responses to $\mathrm{O}_{3}$ exposure}

In addition to the plasma antioxidant moieties outlined above, MDA and protein carbonyl concentrations were also determined, as lipid and protein oxidation markers respectively. Pre-E concentrations for both exposure arms are given below: MDA, $0.35 \pm 0.02$ (air) and $0.44 \pm 0.20$ $\left(\mathrm{O}_{3}\right) \mu \mathrm{M}$; and protein carbonyls $1.6 \pm 1.5$ and $10.1 \pm 15.1$ nmol.mg protein ${ }^{-1}$. As with Pre-E antioxidant concentrations, no significant difference in the concentrations of markers was noted between the two Pre-E values. Neither air nor $\mathrm{O}_{3}$ exposure had any impact upon the concentrations of either marker at any of the time points examined (data not shown).

\section{Antioxidant partition ratios}

Partition ratios (PRs) of antioxidant moieties (UA and AA), determined in both plasma and nasal RTLF, were calculated in order to ascertain whether or not $\mathrm{O}_{3}$ affected the distribution of these moieties between the two

Table 2. - Inflammatory markers in sequential nasal lavage (NL) fluid samples following air and 0.2 parts per million $\mathrm{O}_{3}$ exposure

\begin{tabular}{lccccc}
\hline \multirow{2}{*}{ Parameters } & \multirow{2}{*}{ Exposure } & \multicolumn{4}{c}{ Time point } \\
\cline { 3 - 6 } & & Pre-E & $1 \mathrm{~h}-\mathrm{E}$ & 0h-PE & $1 \mathrm{~h}-\mathrm{PE}$ \\
\hline Neutrophils $10^{4}$ cells & $\mathrm{Air}$ & $1.6(1.0-5.0)$ & $3.1(2.0-4.4)$ & $3.0(0.9-6.6)$ & $0.8(0.4-4.8)^{*}$ \\
& $\mathrm{O}_{3}$ & $0.9(0.7-4.0)$ & $0.8(0.4-5.2)$ & $0.8(0.5-2.7)^{+}$ & $0.9(0.5-3.1)$ \\
MPO $\mu \mathrm{g} \cdot \mathrm{L}^{-1}$ & $\mathrm{Air}$ & $186.2(111.6-346.5)$ & $114.0(48.7-127.4)^{*}$ & $92.8(51.6-146.8)^{*}$ & $60.3(31.2-73.4)^{*}$ \\
& $\mathrm{O}_{3}$ & $90.3(57.7-304.2)$ & $36.2(24.0-161.0)^{*}$ & $63.1(21.9-150.9)^{*}$ & $18.7(13.5-119.3)^{*}$ \\
\hline
\end{tabular}

Data are presented as median with 25 th and 75 th percentiles shown in parentheses. All values are expressed per unit volume of recovered NL fluid $(\mathrm{n}=13)$. Statistical analysis was performed using the Quade two-way (ANOVA) with multiple comparisons using the t-distribution. Rejection of the null hypothesis was assumed at $\mathrm{p}<0.05$. Pre-E: pre-exposure; $1 \mathrm{~h}-\mathrm{E}$ : $1 \mathrm{~h}$ into exposure; $0 \mathrm{~h}-\mathrm{PE}$ : immediately post-exposure; $1 \mathrm{~h}-\mathrm{PE}: 1 \mathrm{~h}$ post-exposure; MPO: myeloperoxidase. ${ }^{*}$ : significantly different from Pre-E value; ${ }^{+}$: significantly different from air value. 

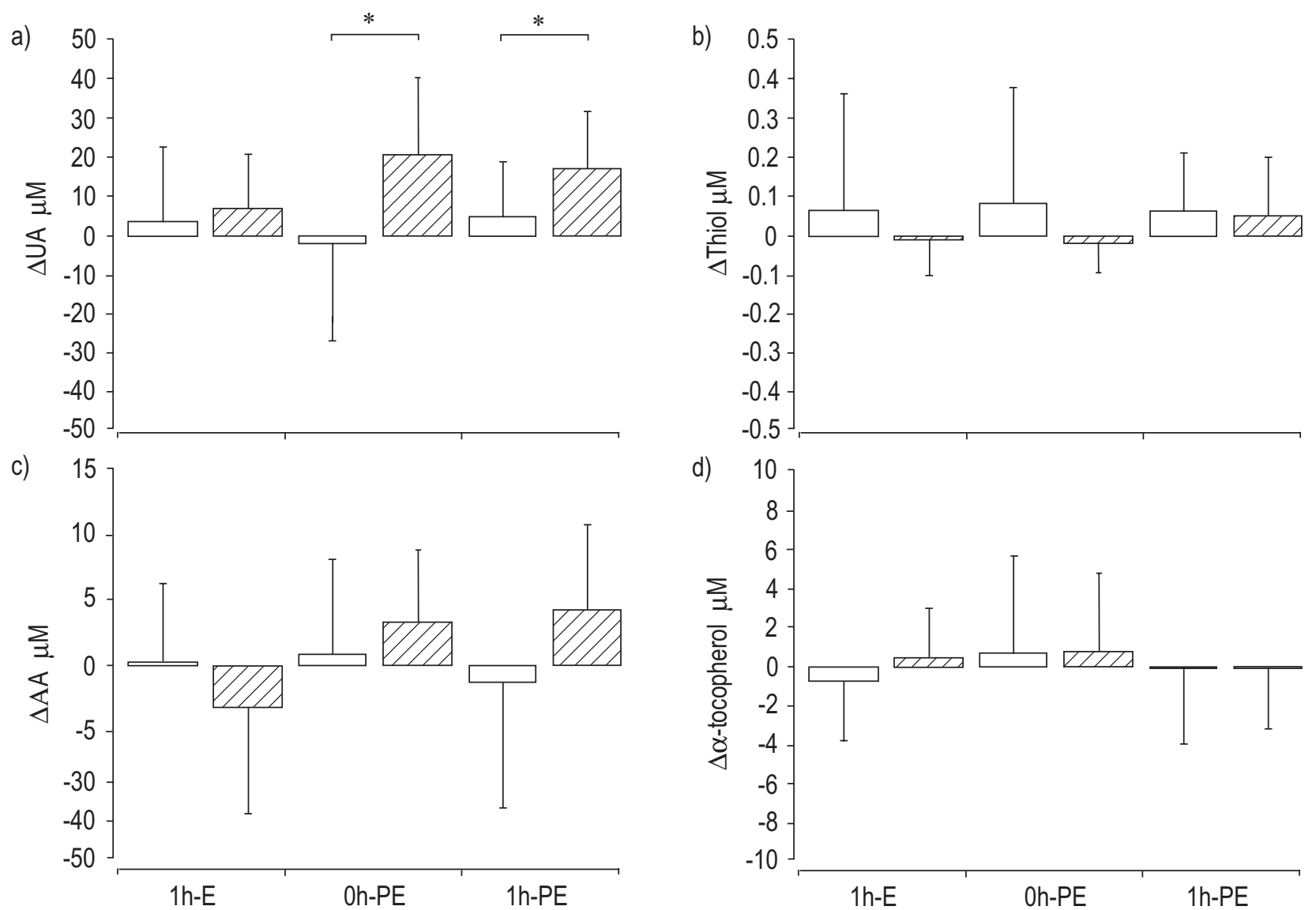

Fig. 2. - Absolute molar concentration changes in plasma antioxidant levels following exposure to 0.2 parts per million $\mathrm{O}_{3}(\mathbb{Z})$ or air $(\square)$ at $1 \mathrm{~h}$ into exposure (1h-E), immediately post-exposure (0h-PE) and $1 \mathrm{~h}$ post-exposure (1h-PE) versus pre-exposure (Pre-E) levels: a) uric acid (UA); b) thiol; c) ascorbic acid (AA); and d) $\alpha$-tocopherol. Data are represented as mean $\pm \mathrm{SD}(\mathrm{n}=13)$. Differences between concentrations at each of the prescribed intervals were assessed using Wilcoxon's signed-rank-test. *: p<0.05.

compartments. The results are summarized in table 3 . The partition ratio of UA closely followed the pattern observed for UA concentration changes in NL, increasing significantly above Pre-E and parallel air control values at $1 \mathrm{~h}-\mathrm{E}$ and $0 \mathrm{~h}-\mathrm{PE}$, the latter occurring despite the compensatory increase in plasma UA concentration at this time point. With respect to the AA PR, increases in both air and $\mathrm{O}_{3}$ exposure arms versus Pre-E values were observed, becoming significant $(\mathrm{p}<0.05)$ at $1 \mathrm{~h}-\mathrm{E}$ in the $\mathrm{O}_{3}$ treated group, and 0h-PE with air. Consequently, a significant difference in AA PR at $1 \mathrm{~h}-\mathrm{E}$ was noted for air versus $\mathrm{O}_{3}$, the $\mathrm{PRs}$ changing by +12.4 (0.6-22.8) after air and $32.8(13.0-50.3)$ after $\mathrm{O}_{3}$ over pre-E values $(\mathrm{p}<0.05)$.

\section{Correlation analyses}

Correlation analyses were performed to determine whether or not effects occurring within nasal RTLFs during air and $\mathrm{O}_{3}$ exposures were predictive of individual symptomatic outcomes. Attention was limited to a consideration of NL fluid and plasma UA responses, as the $\mathrm{O}_{3}$-induced alterations of UA in these two compartments constituted the major finding of this study. Correlations were performed with alterations in pulmonary function and the inflammatory and redox changes observed in differential lavage samples obtained from the same subjects and reported in the accompanying article [12]. Within each

Table 3. - Alterations in blood respiratory tract lining fluid antioxidant partition ratios (PRs) as a consequence of exposure to air or 0.2 parts per million $\mathrm{O}_{3}$

\begin{tabular}{lccccc}
\hline \multirow{2}{*}{ Parameters } & \multirow{2}{*}{ Exposure } & \multicolumn{4}{c}{ Time point } \\
\cline { 3 - 6 } & & Pre-E & 1h-E & 0h-PE & 1 h-PE \\
\hline UA PR $(\mathrm{n}=11)$ & Air & $9.4(4.3-18.2)$ & $9.5(3.6-19.8)$ & $9.3(3.5-23.7)$ & $15.7(4.0-20.0)$ \\
& $\mathrm{O}_{3}$ & $15.0(8.0-18.5)$ & $24.3(15.1-32.5)^{*+}$ & $22.7(13.0-29.6)^{*^{+}}$ & $19.1(14.2-24.4)^{*}$ \\
AA PR $(\mathrm{n}=12)$ & $\mathrm{Air}$ & $10.5(7.2-26.8)$ & $23.6(11.4-36.7)$ & $30.4(14.7-71.8)^{*}$ & $26.3(14.1-60.1)^{*}$ \\
& $\mathrm{O}_{3}$ & $18.6(9.9-23.0)$ & $37.1(31.9-58.8)^{*+}$ & $42.5(32.1-83.7)^{*}$ & $46.1(17.3-67.1)^{*}$ \\
\hline
\end{tabular}

Data are presented as median with 25th and 75th percentiles shown in parentheses. Statistical analysis was performed using the Quade two-way analysis of variance (ANOVA), with multiple comparisons using the t-distribution. Pre-E: pre-exposure; $1 \mathrm{~h}-\mathrm{E}$ : $1 \mathrm{~h}$ into exposure; 0h-PE: immediately post-exposure; $1 \mathrm{~h}-\mathrm{PE}: 1 \mathrm{~h}$ post-exposure; UA: uric acid; AA: ascorbic acid. *: p $<0.05$ versus Pre-E value; ${ }^{+}: \mathrm{p}<0.05$ versus air value. 


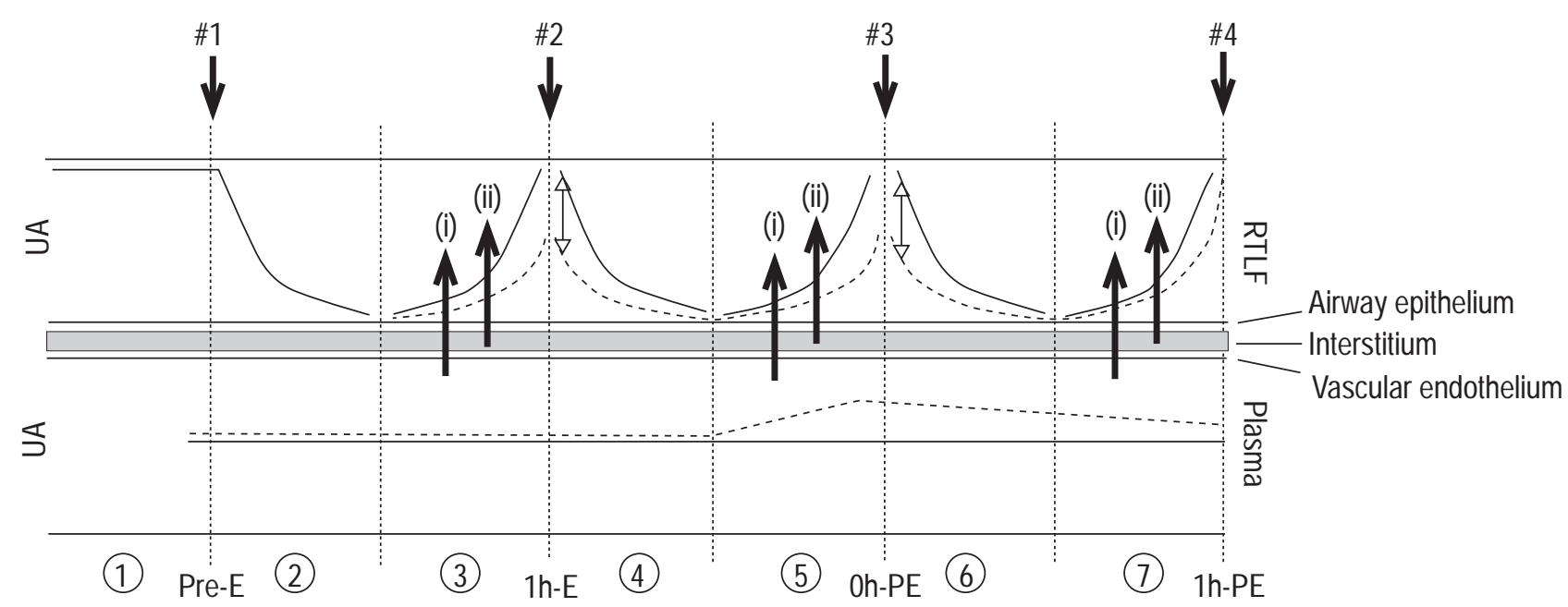

Fig. 3. - Cartoon of washout, oxidative consumption and repletion kinetics of uric acid (UA) in the upper respiratory tract lining fluids (RTLFs) (with corresponding alterations in the plasma concentration) of healthy human subjects exposed to filtered air or 0.2 parts per million $\mathrm{O}_{3}$. 1) Baseline RTLF and plasma UA concentrations, pre-exposure (Pre-E). 2) First blood sample withdrawn and nasal lavage (NL) performed (\#1). This effectively washes out the RTLF; UA concentrations fall to $\sim 20 \%$ of pre-lavage values (unpublished data). 3) First hour of exposure ( - : air; - - - - : $\mathrm{O}_{3}$ ). During this period UA moves into the RTLF from unidentified sources (plasma (i) or cellular stores (ii)) to replace that lost during the first lavage. In the $\mathrm{O}_{3}$-exposed individuals this repletion is opposed by oxidative consumption, such that by the end of the first hour of exposure (1h-E) concentrations in the $\mathrm{O}_{3}$-exposed group are $\sim 60 \%$ of those observed following air, which have recovered back to Pre-E levels. 4) The second plasma sample is taken, the NL procedure performed (\#2). Again, this washes out the RTLFs, effectively creating an identical situation to that observed after the first lavage. 5) Consequently, the recovery response (oxidatively opposed during $\mathrm{O}_{3}$ exposure) during the second hour of exposure mirrors that observed over the first hour, such that the difference between UA concentrations, air versus $\mathrm{O}_{3}\left(\Delta \mathrm{O}_{3}\right)$, should be identical during the two 1-h periods. In addition, a small yet significant increase in plasma UA concentrations is observed. 6) The third blood and nasal lavage samples (\#3) are obtained immediately after the end of the challenge period (0h-PE). This again washes out the majority of the UA from the RTLF. During the next hour (post-exposure), concentrations again recover such that by the final sampling point (\#4) (7), 1 h post-exposure (1h-PE), concentrations have returned to Pre-E values in both groups. Plasma UA remain elevated over Pre-E concentrations.

classification, only those parameters shown to be significantly perturbed by $\mathrm{O}_{3}$ were used: pulmonary function: forced vital capacity, forced expiratory volume in one second, and corrected flow measurements; inflammation: mucosal intercellular adhesion molecule (ICAM)-1 and P-selectin expression, BAL fluid macrophage numbers and macrophage human leukocyte antigen (HLA)-DR expression; and RTLF redox status: bronchial wash (BW) and BAL GSH concentrations, and UA and $\alpha$-tocopherol PRs. This approach reduced the chances of observing spurious interactions.

Correlations were performed using a range of expressions to describe NL UA levels and responses to air and $\mathrm{O}_{3}$ challenge: 1) the overall response of NL UA to $\mathrm{O}_{3}$ (corrected for parallel air effects) (see equations A-F, in Appendix), 2) using Pre-E baseline concentrations (see equation $\mathrm{G}$ ), and 3 ) concentrations after $\mathrm{O}_{3}$, both actual at $1 \mathrm{~h}-\mathrm{E}$, and predicted for $\mathrm{Oh}-\mathrm{PE}$ (see equations $\mathrm{H}-\mathrm{K}$ ). These expressions were then correlated against the significant responses observed in lung function, inflammatory parameters, and proximal and distal airway RTLF redox status, outlined in the previous paragraph of this section. Details of the derivation of these expressions are given in the Appendix. With regard to all the NL UA expressions used, no significant interactions were observed with any of the major symptomatic responses seen in these subjects post$\mathrm{O}_{3}$ challenge. Similarly, upregulation of plasma UA over the interval Pre-E to 0h-PE and 1h-PE was not related to any of the decrements seen in pulmonary function. In contrast, significant interactions were observed with changes in ICAM-1 and differential lavage GSH levels when the change in UA plasma concentration over the interval Pre-E to 1h-PE $\left(\mathrm{O}_{3}\right.$-air) was used: change in $(\Delta)$ ICAM-1: $\rho_{\mathrm{s}}=-0.53, \mathrm{p}=0.03 ; \Delta \mathrm{BW}$ GSH: $\rho_{\mathrm{s}}=0.64, \mathrm{p}=0.01 ; \Delta \mathrm{BAL}$ fluid GSH: $\rho s=0.62, p=0.02$; and $\Delta B A L$ macrophage HLADR expression: $\rho_{\mathrm{s}}=-0.62, \mathrm{p}=0.012$. None of these interactions were apparent over the Pre-E to $0 \mathrm{~h}-\mathrm{PE}$ interval.

\section{Discussion}

The data described in this paper forms part of a larger study, the aim of which was to examine the early symptomatic responses of humans to $\mathrm{O}_{3}$, both in terms of altered pulmonary function and airway RTLF and submucosal responses. It was hypothesized that although the differential lavage samples obtained at 1.5h-PE (described in the accompanying article [12]) allowed events occurring within the airways early following $\mathrm{O}_{3}$ exposure to be visualized, it remained likely that the really pertinent events, those dictating individual symptomatic responses, may occur when $\mathrm{O}_{3}$ is reacting directly with RTLF constituents. The aim of this segment of the study was, therefore, to clarify events occurring within RTLFs during the exposure period itself, and then to attempt to relate these responses to individual $\mathrm{O}_{3}$-induced changes in pulmonary function and airway inflammation.

The major findings of this study were the depletion of nasal RTLF UA and the compensatory increase in plasma UA concentration during the second hour of exposure to $\mathrm{O}_{3}$. Although an initial loss of UA was predictable from previous ex vivo studies [11, 13, 27], it was not clear to what extent in vivo repletion kinetics would mask oxidative consumption by $\mathrm{O}_{3}$. NL fluid UA concentrations were significantly depressed by $\mathrm{O}_{3}$ at $1 \mathrm{~h}-\mathrm{E}$, and remained 
so throughout the exposure period (0h-PE). Notably, no additional loss of UA occurred during the second hour of exposure, indicating that a new pseudo-steady-state concentration had been established. This apparent conservation of UA during the second hour of exposure may have arisen through a variety of mechanisms, including increased synthesis or net movement of UA from other compartments to offset its loss within the RTLF. The finding may, however, simply be an artefact of the repeated sampling procedure employed, as all responses must be viewed in the context of the washout of RTLF constituents by the repeated lavage procedure [28]. With the exception of UA, albumin and total protein, all soluble RTLF components displayed characteristic washout kinetics, suggesting that the rate of repletion of these moieties was not sufficient, at the hourly intervals of sampling used, to replace material washed out by the previous lavage procedure. UA, however, was fully restored to pre-lavage concentrations within $1 \mathrm{~h}$ of RTLF sampling. Consequently, the difference in UA concentration between air and $\mathrm{O}_{3}$ at $1 \mathrm{~h}-\mathrm{E}$ is more correctly viewed as the difference in concentration between that moving freely into the RTLF (air) to replace the UA lost during the first NL and that lost through oxidation by $\mathrm{O}_{3}$. The kinetics of washout, repletion and oxidative consumption of nasal RTLF UA are summarized in figure 3.

The source of nasal RTLF UA, and hence the mechanisms underpinning its high repletion capacity, are not known. Peden et al. [29] demonstrated that NL fluid UA concentrations are increased following cholinergic stimulation of the airways, suggesting that glandular secretion may be important. In support of this, increases in UA levels were found to correlate well with lactoferrin [29], a protein predominately derived from mucosal gland secretions. De novo synthesis of UA within the nasal epithelium seems an unlikely source, as the concentration and activity of xanthine dehydrogenase within the nasal epithelium are low [29]. Also, the time course of repletion is incompatible with such an explanation. Consistent with this low UA synthetic capacity within the nasal environment, repetitive cholinergic stimulation of the nasal epithelium has been shown to diminish the capacity of the submucosal glands to release UA, suggesting that glandular reserves of UA become depleted following repeated stimulation. In the present study, although concentrations of NL fluid UA at 1h-PE did not differ significantly, they were always lower in the $\mathrm{O}_{3}$ exposure arm, suggesting a reduced ability to restore UA levels under this condition. The equivalence between plasma and nasal RTLF UA concentrations (the latter after correction for lavage dilution), allied with the extensive fenestration of the nasal capillary bed and venous sinusoids, has also led to the belief that the majority of RTLF UA may be derived from the plasma pool [30].

The conservation of NL fluid UA during the second hour of $\mathrm{O}_{3}$ exposure was paralleled by a significant increase in the circulating plasma concentration of UA. If RTLF UA were derived from passive movement from the plasma, this increase in plasma UA would effectively establish a new equilibrium between the two pools, permitting more UA to cross into the RTLF. This could then be viewed as an adaptive response, aimed at conserving RTLF UA concentrations. However, no association between individual increases in plasma UA with the respec- tive losses in nasal RTLF UA was observed. This finding may reflect a more complex relationship between the two pools than previously envisioned. Indeed, regional variations in airway permeability [31] may target increased plasma UA to the more distal RTLFs. For example, in the accompanying article [12], significantly altered PRs between plasma and BAL fluid UA concentrations at $1.5 \mathrm{~h}-$ $\mathrm{PE}$ are reported, whereas initial differences in plasma/NL fluid PRs are no longer evident by this time. Movement of UA into the lower airways $18 \mathrm{~h}$ after exposure to 400 parts per billion $\mathrm{O}_{3}$ has been reported [32]. Furthermore, $\mathrm{NO}_{2}$ inhalation results in a transient decrease in distal RTLF UA concentrations followed by significant elevation above pre-E values 6 and $24 \mathrm{~h}$ post-exposure [23]. Taken together, these two observations suggest that increased secretion and/or passive movement of UA into the airways may represent a common mechanism for ameliorating the effects of oxidative air pollutants.

A number of explanations for the increased plasma UA pool are possible, but each imply that a systemic response is elicited by oxidative events conceptually confined to the RTLFs. An increased rate of purine metabolism, via either the increased expression or activity of xanthine dehydrogenase, constitutes one possible mechanism. Although epithelial cells have been reported to be rich sources of xanthine dehydrogenase/oxidase, previous studies have revealed the nasal epithelium to contain only modest concentrations of this enzyme [29]. Therefore, if increased synthesis of UA underlies this effect, the site of production is unlikely to be the immediate cellular interface. Consequently, the changes occurring within nasal RTLFs would have to be transmitted beyond this extracellular environment. Although $\mathrm{O}_{3}$ has been demonstrated to exhibit a range of extrapulmonary effects in animal models $[33,34]$, the underlying mechanisms and/or mediators of such remote responses are not known. PRYOR et al. [7] have suggested that lipid ozonation products may function in this manner as a consequence of their relatively long half-lives and structural similarities to recognized signalling molecules. However, in the present study, no evidence of lipid oxidation, determined via increases in the concentration of MDA, was detected. Circulating UA concentrations could also be raised by increased renal reabsorption or the release of cellular stores of UA. Although there is no evidence within the literature to support the former contention, the response of mucosal glands to cholerinergic stimulation would suggest that these cells can be induced to release stored UA.

Previous ex vivo studies using BAL and NL to sample RTLF antioxidants have shown that exposure to $\mathrm{O}_{3}$ results in a marked depletion of AA and, to a lesser extent, GSH $[11,13]$. Although the present study demonstrated that $\mathrm{O}_{3}$ exposure resulted in a marked loss of UA and a small loss of GSH in vivo, the absence of AA consumption was surprising. This may be due to the dramatic depletion of NL AA that occurred through washout alone, a change that may have overshadowed any additional $\mathrm{O}_{3}$-dependent loss. Additionally, AA concentrations in nasal RTLFs are only $5-10 \%$ of those of UA, and although their absolute reactivities toward $\mathrm{O}_{3}$ are similar [35], it would be expected that consumption of UA would predominate. Further, although in the current study NL fluid AA concentrations remained unaltered by $\mathrm{O}_{3}$, an increase in the PR was observed at $1 \mathrm{~h}-\mathrm{E}$. This finding may reflect a 
very early adaptive response to replenish RTLF AA lost prior to the first sampling time point, implying that even within the exposure period itself adaptive responses to the oxidative insult are occurring.

Although attempts were made to relate the UA responses observed in nasal RTLF to the symptomatic responses (see accompanying article [12]), in no case were any significant associations found. This has been taken to suggest that UA loss from nasal RTLF was not functionally related to any of these symptomatic outcomes. In contrast, the adaptive increase in plasma UA concentrations following $\mathrm{O}_{3}$ exposure appeared to be related to a number of inflammatory and redox parameters. Those subjects displaying the greatest increases in plasma UA at $1 \mathrm{~h}-\mathrm{PE}$ also displayed the smallest upregulation of ICAM1 expression and alveolar macrophage activation, suggesting that the inflammatory response to $\mathrm{O}_{3}$ was reduced in these individuals. In addition, those subjects with the greatest increases in plasma UA also displayed the largest increase in BW and BAL fluid GSH at 1.5h-PE. Consequently, the absolute change in redox status of the RTLF, as a consequence of $\mathrm{O}_{3}$ exposure, is not the critical determinant of response. Rather, the capacity to mount an adaptive response to the initial insult is more important, although the precise nature of this or how it is regulated requires further investigation.

The results of the present study advance our understanding of the extracellular antioxidant screen within the upper respiratory tract and its response to environmentally encountered $\mathrm{O}_{3}$ concentrations. It has been demonstrated that nasal respiratory tract lining fluid uric acid is consumed by $\mathrm{O}_{3}$ in a manner consistent with it conferring a protective function in this environment. Furthermore, an adaptive response to $\mathrm{O}_{3}$ was observed with increases in plasma uric acid. Whilst uric acid may confer protection locally, the role of upper airway uric acid as a sink for inhaled $\mathrm{O}_{3}$, limiting the dose penetrating to the distal lung and hence reducing individual symptomatic responses, is not supported by these findings. Rather, the capacity to mount an adaptive antioxidant response to the initial insult appeared critical.

\section{Appendix: Range of response and concentration expressions used to describe the action of ozone on NL fluid $U A$ in the correlation analysis}

\section{Effect expressions weighted for pre-exposure values}

A)

$$
\begin{aligned}
& \Delta \mathrm{O}_{3}(1 \mathrm{~h})=\left([\mathrm{UA}] 1 \mathrm{~h}-\mathrm{E}, \mathrm{O}_{3}-[\mathrm{UA}]{\text { PreE }, \mathrm{O}_{3}}\right) \\
& -([\mathrm{UA}] 1 \mathrm{~h}-\mathrm{E}, \mathrm{air}-[\mathrm{UA}] \text { PreE,air })
\end{aligned}
$$

B)

$$
\begin{aligned}
& \Delta \mathrm{O}_{3}(2 \mathrm{~h})=\left([\mathrm{UA}] 0 \mathrm{~h}-\mathrm{PE}, \mathrm{O}_{3}-[\mathrm{UA}] \mathrm{PreE}_{\mathrm{O}} \mathrm{O}_{3}\right) \\
& -([\mathrm{UA}] 0 \mathrm{~h}-\mathrm{PE}, \mathrm{air}-[\mathrm{UA}] \text { PreE,air })
\end{aligned}
$$

C)

$$
\Delta \mathrm{O}_{3} \overline{\mathrm{x}}_{(1 \mathrm{~h})}=\frac{(\mathrm{A}+\mathrm{B})}{2}
$$

Effect expressions unweighted for pre-exposure values

D)

E)

$$
\Delta \mathrm{O}_{3}(1 \mathrm{~h})[\mathrm{UA}] 1 \mathrm{~h}-\mathrm{E}, \mathrm{O}_{3}-[\mathrm{UA}] 1 \mathrm{~h}-\mathrm{E}, \text { air }
$$

$\Delta \mathrm{O}_{3}(2 \mathrm{~h})=[\mathrm{UA}] 0 \mathrm{~h}-\mathrm{PE}, \mathrm{O}_{3}-[\mathrm{UA}] \mathrm{oh}-\mathrm{PE}$, air

F)

$\Delta \mathrm{O}_{3} \overline{\mathrm{x}}(1 \mathrm{~h})=\frac{\mathrm{D}+\mathrm{E}}{2} \quad$ (average loss over the two $1-\mathrm{h}$ periods)

\section{Concentration correlates}

G)

$$
[\mathrm{UA}] \overline{\mathrm{X}} \mathrm{Pre}-\mathrm{E})=\frac{[\mathrm{UA}] \operatorname{Pre}-\mathrm{E}, \mathrm{air}+[\mathrm{UA}] \mathrm{Pre}-\mathrm{E}, \mathrm{O}_{3}}{2}
$$

$\mathrm{H})$

$$
[\mathrm{UA}] \overline{\mathrm{X}}(1 \mathrm{~h}-\mathrm{E}, \mathrm{air})=\frac{[\mathrm{UA}] 1 \mathrm{~h}-\mathrm{E}, \mathrm{air}+[\mathrm{UA}] 0 \mathrm{~h}-\mathrm{PE}, \mathrm{air}}{2}
$$

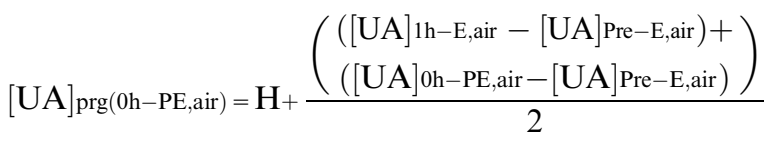

J)

$$
[\mathrm{UA}] \overline{\mathrm{x}}\left(1 \mathrm{~h}-\mathrm{E}, \mathrm{O}_{3}\right)=\frac{[\mathrm{UA}] 1 \mathrm{~h}-\mathrm{E}, \mathrm{O}_{3}+[\mathrm{UA}] 0 \mathrm{~h}-\mathrm{PE}, \mathrm{O}_{3}}{2}
$$

K)

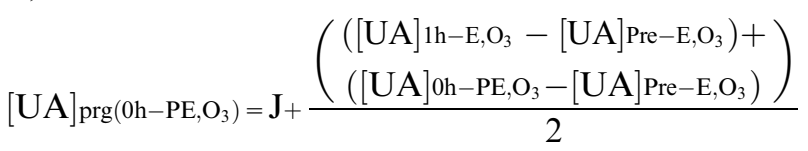

Full correlation analysis. All of the equations outlined above were used in the full correlation analysis. UA responses were always corrected for the parallel air response and were expressed either as the change in concentration over the first hour of exposure $\left(\Delta \mathrm{O}_{3}(\mathrm{hh})\right)$, the change at $2 \mathrm{~h}\left(\Delta \mathrm{O}_{3}(2 \mathrm{~h})\right)$, or the average change over the successive hourly intervals $\left(\Delta \mathrm{O}_{3} \overline{\mathrm{x}}(1 \mathrm{~h})\right)$. Responses, expressed in terms of the concentration differences seen at each of the sampling intervals $\left(\mathrm{O}_{3}-\right.$ air $)$ were either weighted for the pre-air and $\mathrm{O}_{3}$ exposure concentration (Pre$\mathrm{E}, \mathrm{O}_{3}$ and Pre-E,air) as in equations $\mathrm{A}-\mathrm{C}$, or not, as in equations $\mathrm{D}-\mathrm{F}$. The latter expressions were used for correlations with parameters determined in BAL fluid where pre-E values were not available. UA concentration at $1 \mathrm{~h}$ post-air and post- $\mathrm{O}_{3}$ exposure (1h-E, air and $\left.1 \mathrm{~h}-\mathrm{E}, \mathrm{O}_{3}\right)$; at $2 \mathrm{~h}$, or immediately post air and $\mathrm{O}_{3}$ exposure (0h-PE,air and $\mathrm{Oh}-\mathrm{PE}, \mathrm{O}_{3}$ ). The average baseline UA concentration (xPre-E) was determined from the pre-exposure air and $\mathrm{O}_{3}$ concentrations; equation $\mathrm{G}$. The average concentration of RTLF_UA remaining after $1 \mathrm{~h}$ exposure to air or $\mathrm{O}_{3}$ ([UA] $\bar{x}\left(1 \mathrm{~h}-\mathrm{E}\right.$, air) and $[\mathrm{UA}] \overline{\mathrm{x}}\left(1 \mathrm{~h}-\mathrm{E}, \mathrm{O}_{3}\right)$ ) was calculated from the concentrations observed at $1 \mathrm{~h}-\mathrm{E}$ and $0 \mathrm{~h}-\mathrm{PE}$, as these two concentrations represented the outcome of two repeated responses; equations $\mathrm{H}$ and J. Finally, the projected concentration of UA which would have remained 
immediately post exposure, had repletion not offset this loss was detemined for air ([UA]prg(Oh-PE,air)) and $\mathrm{O}_{3}$ ([UA]prg $\left.\left(0 \mathrm{~h}-\mathrm{PE}, \mathrm{O}_{3}\right)\right)$ in equations I and $\mathrm{K}$, respectively. These two expressions take the average concentration observed after a single hours exposure (as outlined before) and add to it average loss observed over the $1 \mathrm{~h}-\mathrm{E}$ and $0 \mathrm{~h}-\mathrm{PE}$ intervals.

\section{References}

1. Slade R, Crissman K, Norwood J, et al. Comparison of antioxidant substances in bronchoalveolar lavage cells and fluid from humans, guinea pigs, and rats. Exp Lung Res 1993; 19: 469-484.

2. Peden DB, Hohman R, Brown ME, et al. Uric acid is a major antioxidant in human nasal airway secretions. Proc Natl Acad Sci USA 1990; 87: 7638-7642.

3. Heffner JE, Repine JE. Antioxidants and the lung. In: Crystal RG, West JB, eds. The Lung: Scientific Foundations. New York, Raven Press, 1992; pp. 1811-1820.

4. Pryor WA. How far does ozone penetrate into the pulmonary air/tissue boundary before it reacts? Free Rad Biol Med 1992; 12: 83-88.

5. Langford SD, Bidani A, Postlethwait EM. Ozone-reactive absorption by pulmonary epithelial lining fluid constituents. Toxicol Appl Pharmacol 1995; 132: 122-130.

6. Postlethwait EM, Langford SD, Jacobson LM, et al. $\mathrm{NO}_{2}$ reactive absorption substrates in rat pulmonary surface lining fluids. Free Rad Biol Med 1995; 19: 553-563.

7. Pryor WA, Squadrito GL, Friedman M. The cascade mechanism to explain ozone toxicity: the role of lipid ozonation products. Free Rad Biol Med 1995; 19: 935-941.

8. Cantin AM, North SL, Hubbard RC, et al. Normal alveolar epithelial lining fluid contains high levels of glutathione. J Appl Physiol 1987; 63: 152-157.

9. Gerrity TR, Weaver RA, Berntsen J, et al. Extrathoracic and intrathoracic removal of ozone in tidal breathing of humans. J Appl Physiol 1988; 65: 393-400.

10. Cross CE, Matchnik PA, Kaur H, et al. Effect of ozone on human plasma. FASEB J 1991; 5: A886.

11. Mudway IS, Housley D, Eccles R, et al. Differential depletion of human respiratory tract antioxidants in response to ozone challenge. Free Rad Res 1996; 25: 499-513.

12. Blomberg A, Mudway IS, Nordenhä UC, et al. Ozoneinduced lung function decrements do not correlate with early airway inflammatory or antioxidant responses. Eur Respir J 1999; 13: 1418-1428.

13. Housley DG, Muclway I, Kelly FJ, et al. Depletion of urate in human nasal lavage following in vitro ozone exposure. Int J Biochem Cell Biol 1995; 25: 1153-1159.

14. Krishna MT, Blomberg A, Biscione GL, et al. Short-term ozone exposure upregulates P-selectin in normal human airways. Am J Respir Crit Care Med 1997; 155: 17981803.

15. Harder S, Peden DB, Koren HS, et al. Comparison of nasal lavage techniques for analysis of nasal inflammation. Am J Respir Crit Care Med 1994; 151: A565.

16. Baker MA, Cerniglia GJ, Zaman A. Microtiter plate assay for the measurement of glutathione and glutathione disulfide in large numbers of biological samples. Anal Biochem 1990; 190: 360-365.

17. Iriyama $\mathrm{K}$, Yoshiura $\mathrm{M}$, Iwamoto $\mathrm{T}$, et al. Simultaneous determination of uric and ascorbic acids in human serum by reversed-phase high-performance liquid chromatography with electrochemical detection. Anal Biochem 1984; 141: 238-243.

18. Bieri JG, Tolliver TJ, Catignani GL. Simultaneous determination of alpha-tocopherol and retinol in plasma or red cells by high pressure liquid chromatography. Am J Clin Nutr 1979; 32: 2143-2149.

19. Waynor DD, Barton GW, Ingold KU, et al. The relative contributions of vitamin E, urate, ascorbate and proteins to the total peroxyl radical trapping antioxidant activity of human blood plasma. Biochem Biophys Acta 1987; 924: $408-419$.

20. Karlsson K, Marklund SL. Plasma clearance of human extracellular-superoxide dismutase $\mathrm{C}$ in rabbits. $J$ Clin Invest 1988; 82: 762-766.

21. Smith PK, Krohn RI, Hermanson GT, et al. Measurement of protein using bicinchoninic acid. Anal Biochem 1985; 150: 76-85.

22. Chirico S. High-performance liquid chromatographybased thiobarbituric acid tests. Methods Enzymol 1994; 233: 314-318.

23. Kelly FJ, Blomberg A, Frew AJ, et al. Antioxidant kinetics in lung lining fluid following exposure of human to nitrogen dioxide. Am J Respir Crit Care Med 1996; 154: 1700-1705.

24. Reznick AZ, Packer L. Oxidative damage to proteins: spectrophotometric method for carbonyl assay. Methods Enzymol 1994; 233: 357-363.

25. Mudway I, Blomberg A, Frew AJ, et al. Intra-individual antioxidant measurements in nasal lavage fluid from nonsmoking normal subjects. Am J Respir Crit Care Med 1997; 155: A191.

26. Stocker R, Frei B. Endogenous antioxidant defences in human blood plasma. In: Sies H, ed. Oxidative Stress: Oxidants and Antioxidants. San Diego, Academic Press, 1991: 213-243.

27. Housley DG, Mudway I, Kelly FJ, et al. Depletion of urate in human nasal lavage following in vitro ozone exposure. Int J Biochem Cell Biol 1995; 27: 1153-1159.

28. Merrill W, O'Hearn E, Rankin J, et al. Kinetic analysis of respiratory tract proteins recovered during a sequential lavage protocol. Am Rev Respir Dis 1982; 126: 617-620.

29. Peden DB, Swiersz M, Ohkubo K, et al. Nasal secretion of the ozone scavenger uric acid. Am Rev Respir Dis 1993; 148: 455-461.

30. Eccles R. A role for the nasal cycle in respiratory defence. Eur Respir J 1996; 9: 371-376.

31. Widdicombe J. Why are the airways so vascular? Thorax 1993; 48: 290-295.

32. Koren HS, Hatch GE, Graham DE. Nasal lavage as a tool in assessing acute inflammation in response to inhaled pollutants. Toxicology 1990; 60: 15-25.

33. Kelly FJ, Birch S. Ozone exposure inhibits cardiac protein synthesis in the mouse. Free Rad Biol Med 1993; 14: 443-446.

34. Raman I, Massaro GD, Massaro D. Exposure of rats to ozone: evidence of damage to heart and brain. Free Rad Biol Med 1991; 12: 323-326.

35. Giamalva DH, Church DF, Pryor WA. A comparison of the rates of ozonation of biological antioxidants and oleate and linoleate esters. Biochem Biophys Res Commun 1985; 133: 773-779. 